With compliments of the Author 


\title{
New Synthesis of 2,3-Diarylxanthones
}

\author{
Clementina M. M. Santos, ${ }^{\mathrm{a}, \mathrm{b}}$ Artur M. S. Silva, ${ }^{* b}$ José A. S. Cavaleiro ${ }^{\mathrm{b}}$ \\ a Department of Agro-Industries, Escola Superior Agrária de Bragança, Campus de Santa Apolónia, 5301-855 Bragança, Portugal \\ b Department of Chemistry, University of Aveiro, Campus Universitário de Santiago, 3810-193 Aveiro, Portugal \\ Fax +351(234)370084; E-mail: arturs@dq.ua.pt \\ Received 23 September 2005
}

\begin{abstract}
A new synthesis for 2,3-diarylxanthones is described. This was accomplished by aldol condensation of 3-bromo-2-methylchromone with benzaldehydes leading to the formation of 3-bromo-2-styrylchromones, followed by Heck reaction with styrenes.
\end{abstract}

Key words: diarylxanthones, 2-styrylchromones, Heck reaction, 3-bromo-2-methylchromone, electrocyclisation

Xanthones (dibenzo- $\gamma$-pyrones) are a group of heterocyclic compounds, which are widely distributed in Guttifer$a e$ and Gentianaceae families. However, as aglycone derivatives, they also occur in six other families and xanthone- $C$-glycosides are widespread among angiosperms. ${ }^{1}$

Due to their potential biological properties, compounds with a xanthone core are of great interest for chemists, biologists and pharmacologists. Natural and synthetic derivatives have shown important anti-microbial, anti-tumour, anti-inflammatory as well as antioxidant activities. ${ }^{1,2}$

The biogenesis of xanthones largely dictates the nature and position of substituents. The development of new synthetic routes extends the possibilities of having other types of substituted xanthones for biological assessment and for the rationalisation of structure-activity relationships. In the present communication we report a new synthetic route to novel 2,3-diarylxanthones. To the best of our knowledge, xanthones bearing aryl substituents are scarce and no natural or synthetic xanthones have been reported with a 2,3-diaryl substitution pattern. A European Patent ${ }^{3}$ describes the use of 2-phenylxanthone as solvent in the synthesis of aromatic polyketones whereas Kelkar et al. ${ }^{4}$ reported the synthesis of 3-phenylxanthone derivatives from the Diels-Alder reactions of 2-styrylchromones with substituted enamines.

Several methods have been extensively described in literature for the synthesis of xanthones. The traditional synthetic route involves the bonding of two benzene rings through a pyran unit. The carbonyl group can be introduced by a Friedel-Crafts acylation or a Fries rearrangement, among others. However, these procedures usually present low yields and a range of secondary reactions. ${ }^{5}$

We report here a new synthesis of several novel 2,3-diarylxanthones, starting from 2'-hydroxyacetophenone (Schemes 1-3).

SYNLETT 2005, No. 20, pp 3095-3098

Advanced online publication: 28.11.2005

DOI: 10.1055/s-2005-921928; Art ID: D29105ST

(c) Georg Thieme Verlag Stuttgart · New York
3-Bromo-2-methylchromone (4) was prepared in good overall yield according to the sequences shown in Scheme 1. In the three-step sequence, known as the Baker-Venkataraman ${ }^{6}$ method, we performed the acetylation of 2'-hydroxyacetophenone (1) followed by treatment with sodium hydride or potassium tert-butoxide, in refluxing dry THF, leading to 1,3-diketone $\mathbf{3}$ (via intramolecular Claisen condensation). A modified BakerVenkataraman process proposed by Ares et al., ${ }^{7}$ involving a one-pot synthesis, was also applied to the formation of the 1,3-diketone $3(80 \%)$. Bromination and cyclisation of this 1,3-diketone 3 into the desired 3-bromo-2-methylchromone (4) was achieved in an one-pot synthesis, using 1.5 mol of bromine in ethanol followed by acidification. ${ }^{8}$

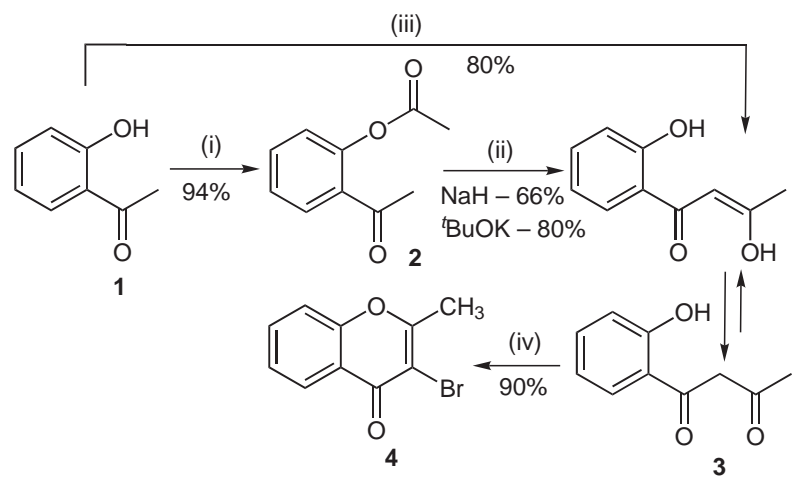

Scheme 1 Reagents and conditions: (i) $\mathrm{CH}_{3} \mathrm{COCl}$, pyridine, r.t., 15 $\mathrm{h}$; (ii) $\mathrm{NaH}$ or $t$-BuOK, THF, reflux, $2 \mathrm{~h}$; (iii) $1 . \mathrm{CH}_{3} \mathrm{COCl}, t$-BuOK, THF, r.t., 2 h; 2. $t$-BuOK, reflux, 2 h; (iv) 1. $\mathrm{Br}_{2}$, EtOH, r.t., 2 h; 2. $\mathrm{HCl}$, reflux, $2 \mathrm{~h}$

Several methods have been applied to the synthesis of 2styrylchromones; one of which involves the condensation of 2-methylchromones with benzaldehydes. ${ }^{9}$ Extension of this methodology to the reaction of 3-bromo-2-methylchromone (4) with benzaldehydes $\mathbf{5 a}, \mathbf{b}$ allows access to 3bromo-2-styrylchromones 6a,b (Scheme 2). ${ }^{10,11}$ We have found that it is necessary to use an excess (4 molar equiv) of base and extended reaction times to obtain the best yields (Table 1). The low yield of 3-bromo-2-styrylchomone $\mathbf{6 b}$ when compared with that of compound $\mathbf{6 a}$, can be explained by considering the lower reactivity of the 4-benzyloxybenzaldehyde (5b), due to the presence of an electron-donating group in the 4-position. ${ }^{12}$

Over the last three decades, palladium-catalysed coupling of olefins with aryl- or vinyl halides (the Heck reaction) has become one of the most versatile methods for $\mathrm{C}-\mathrm{C}$ 
<smiles>[R]c1ccc(/C=C/c2oc3ccc(C#Cc4ccc([10B])cc4)cc3c(=O)c2Br)cc1</smiles>

Scheme 2 Reagents and conditions: (i) $\mathrm{NaOMe}, \mathrm{MeOH}$, r.t., $48 \mathrm{~h}$

Table 1 Condensation of 3-Bromo-2-methylchromone (4) with Benzaldehydes 5a,b

\begin{tabular}{llll}
\hline Base (equiv) & Time (h) & $\mathbf{6 a}(\%)$ & $\mathbf{6 b}(\%)$ \\
\hline 4 & 2 & 55 & 52 \\
4 & 12 & 56 & 52 \\
4 & 24 & 61 & 53 \\
4 & 48 & 87 & 68 \\
4 & 72 & 49 & 49 \\
\hline
\end{tabular}

bond formation in organic synthesis. ${ }^{13}$ In our work, the olefins are the styrenes $\mathbf{7 a - c}$ and the bromine derivatives are the 3-bromo-2-styrylchromones 6a,b (Scheme 3). ${ }^{14}$

In the first instance, we applied the Heck conditions to the reaction of 3-bromo-2-styrylchromone $\mathbf{6 a}$ with styrene $7 \mathbf{a}$ (Scheme 3). TLC analysis of the reaction mixture revealed the presence of a major and a minor product. The NMR spectrum of the main product indicates that we had the 2,3-diphenylxanthone $\mathbf{8 a},{ }^{15}$ and not the expected 2,3distyrylchromone 9a. The analysis of the NMR spectrum of the minor compound revealed the presence of 2,3diphenyl-3,4-dihydroxanthone $\mathbf{1 0 a},{ }^{16}$ a semi-oxidized intermediate of the final xanthone. This fact indicates that the Heck reaction leads to the formation of the 2,3distyrylchromone 9a, and probably the high reaction temperature promotes the electrocyclisation followed by oxidation, to give the 2,3-diphenylxanthone 8a. The same type of thermal electrocyclisation has been already reported ${ }^{17}$ while the further oxidation is promoted by the aromaticity of the final compound.

When we applied the same Heck conditions to the reaction of $\mathbf{6 a}, \mathbf{b}$ with other styrenes similar results were obtained in each case: a major product corresponding to 2,3diarylxanthones $8 \mathrm{~b}-\mathbf{f}$ and the minor product, corresponding to the semi-oxidized products $\mathbf{1 0 b}-\mathbf{f}$, were obtained.

The formation of compounds 10a-f can be explained by the mechanism depicted in Scheme 3. The Heck reaction of $\mathbf{6 a}, \mathbf{b}$ with styrenes $7 \mathbf{a}-\mathbf{c}$ leads to the formation of the 2,3-distyrylchromones 9a-f which undergo in situ electrocyclisation to give 2,3-diaryl-2,3-dihydroxanthones 11a-f and these compounds are converted into 2,3-diphenyl-3,4-dihydroxanthones 10a-f by a [1,5] sigmatropic hydrogen migration. This [1,5] sigmatropic hydrogen shift is facilitated by the resonance stabilisation of the

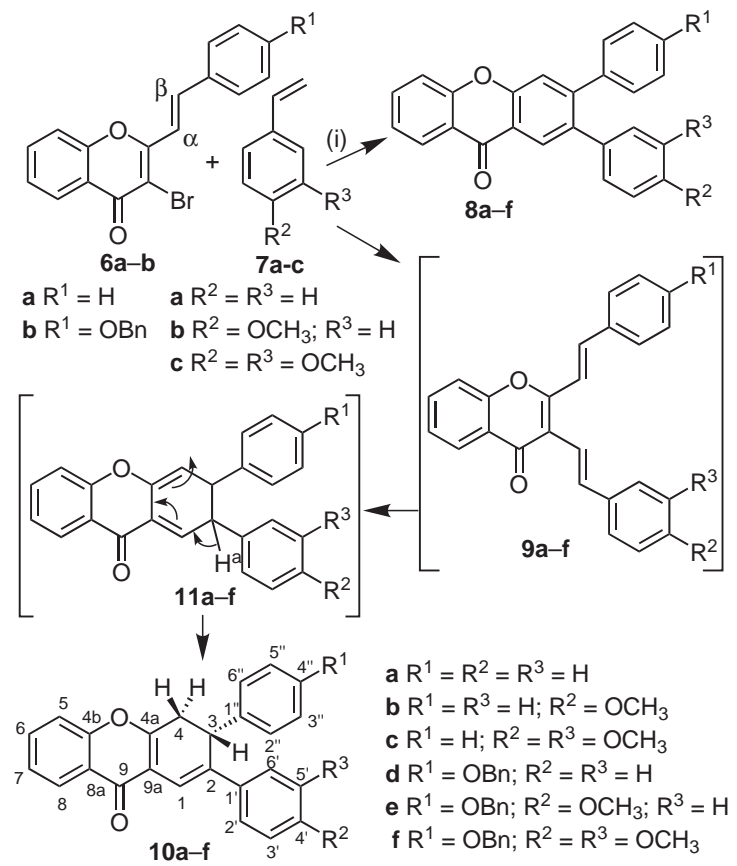

Scheme 3 Reagents: (i) $\mathrm{Et}_{3} \mathrm{~N}, \mathrm{Pd}\left(\mathrm{PPh}_{3}\right)_{4}, \mathrm{PPh}_{3}, \mathrm{NMP}$

chromone nucleus and by the acidity of $\mathrm{H}^{\mathrm{a}}$ of compounds 11a-f due to the resonance with carbonyl group.

After this study, an extensive investigation into the coupling reaction conditions of 3-bromo-2-styrylchromones 6a,b with styrenes $7 \mathbf{a}-\mathbf{c}$ was performed..$^{18}$ This involved varying the amount and type of base, phosphine and catalyst and also several solvents, temperature and reaction time; the best obtained yields being accomplished with the conditions described in Table 2.

Table 2 Heck Reaction of 3-Bromo-2-styrylchromones $\mathbf{6 a}, \mathbf{b}$ with Styrenes $\mathbf{7 a}-\mathbf{c}$

\begin{tabular}{llllll}
\hline Comp & $\begin{array}{l}\mathrm{Et}_{3} \mathrm{~N} \\
(\text { equiv) }\end{array}$ & $\begin{array}{l}\text { Catalyst } \\
(\text { equiv) }\end{array}$ & $\mathbf{7 a - c}$ & Conditions $^{\mathrm{b}}$ & Yield (\%) \\
\hline $\mathbf{8 a}$ & 4 & 0.05 & 5 & $160{ }^{\circ} \mathrm{C}, 6 \mathrm{~h}$ & 56 \\
$\mathbf{8 b}$ & 4 & 0.05 & 5 & Reflux, 3 h & 49 \\
$\mathbf{8 c}$ & 1 & 0.05 & 2 & $160{ }^{\circ} \mathrm{C}, 12 \mathrm{~h}$ & 45 \\
$\mathbf{8 d}$ & 1 & 0.05 & 5 & Reflux, 6 h & 51 \\
$\mathbf{8 e}$ & 4 & 0.05 & 5 & Reflux, 6 h & 66 \\
$\mathbf{8 f}$ & 1 & 0.05 & 5 & Reflux, 3 h & 20 \\
\hline
\end{tabular}

${ }^{a}$ Catalyst: $\mathrm{Pd}\left(\mathrm{PPh}_{3}\right)_{4}$.

${ }^{\mathrm{b}}$ In all cases 0.1 molar equiv of $\mathrm{PPh}_{3}$ were used.

All the synthesized compounds have been characterized by NMR, MS and elemental analysis. The most important feature in the ${ }^{1} \mathrm{H}$ NMR spectra of 3-bromo-2-styrylchromones $\mathbf{6 a}, \mathbf{b}$ are the resonances assigned to vinylic protons $\mathrm{H}-\beta(\mathrm{d}, J=15.8-16.0 \mathrm{~Hz} ; \delta=7.73,7.62 \mathrm{ppm})$ that appear at higher frequency values than those of $\mathrm{H}-\alpha(\mathrm{d}$, $J=15.8-16.0 \mathrm{~Hz} ; \delta=7.51,7.30 \mathrm{ppm})$ due to the mesom- 
eric deshielding effect of the carbonyl group. The coupling constants ${ }^{3} J_{\mathrm{H} \alpha-\mathrm{H} \beta}=$ ca. $16 \mathrm{~Hz}$ indicates a transconfiguration for such vinylic systems. The main characteristics in the ${ }^{1} \mathrm{H}$ NMR spectra of 2,3-diarylxanthones ${ }^{15,19}$ 8a-f are the resonances of $\mathrm{H}-1$ and $\mathrm{H}-4$, which appear as two singlets, at $\delta=8.32-8.38 \mathrm{ppm}$ and $\delta=7.52-7.57 \mathrm{ppm}$, respectively. The identification of the structures of the intermediate 2,3-diaryl-3,4-dihydroxanthones 10a-f was possible by the analyses of their HMBC and NOESY spectra (Figure 1). In the HMBC spectra of 3,4-dihydroxanthones 10a-f it was possible to observe the connectivity between protons $\mathrm{H}-1$ (singlet) and $\mathrm{H}-8$ (double doublet) with carbon C-9. In the NOESY spectrum of compound $10 \mathbf{f}^{20}$ it was possible to observe the close proximity of $\mathrm{H}-1$ with $\mathrm{H}-2^{\prime}$ and $\mathrm{H}-6^{\prime}$ and of $\mathrm{H}-3$ with $\mathrm{H}-2^{\prime}$, $\mathrm{H}-6^{\prime}, \mathrm{H}-2^{\prime \prime} 6^{\prime \prime}$ and $\mathrm{H}-4$ cis and $\mathrm{H}-4$ trans with $\mathrm{H}-2^{\prime \prime} 6$ ". ${ }^{21}$

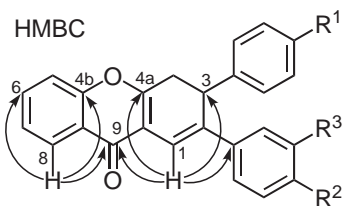

$10 a-f$

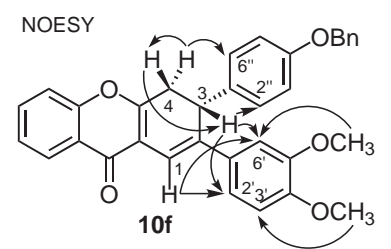

Figure 1 Main results obtained in the HMBC (connectivities) and NOESY (close proximity) spectra of compounds $\mathbf{1 0}$

In conclusion, we have established a new route for the synthesis of novel 2,3-diarylxanthones 8a-f. The condensation reaction of 3-bromo-2-methylchromone (4) with benzaldehydes $\mathbf{5 a}, \mathbf{b}$ led to the formation of new derivatives of 2-styrylchromone $\mathbf{6 a}, \mathbf{b}$. The Heck type reaction of these derivatives with styrenes $7 \mathbf{a}-\mathbf{c}$ provides the novel 2,3-diarylxanthones 8a-f and 2,3-diaryl-3,4-dihydroxanthones 10a-f.

\section{Acknowledgment}

Thanks are due to the University of Aveiro, 'Fundação para a Ciência e a Tecnologia' and FEDER for funding the Organic Chemistry Research Unit and the project POCTI/QUI/38394/2001. One of us (C. M. M. Santos) is also grateful to PRODEP 5.3 for financial support.

\section{References}

(1) (a) Hostettman, K.; Hostettman, M. In Methods in Plant Biochemistry: Plant Phenolics; Dey, P. M.; Harbone, J. B., Eds.; Academic Press: New York, 1989, Vol. 1, 493.

(b) Bennett, G. J.; Lee, H.-H. Phytochemistry 1989, 28, 967.

(2) (a) Abdel-Lateff, A.; Klemke, C.; König, G. M.; Wright, A. D. J. Nat. Prod. 2003, 66, 706. (b) Chiang, Y.-M.; Kuo, Y.H.; Oota, S.; Fukuyama, Y. J. Nat. Prod. 2003, 66, 1070. (c) Pedro, M.; Cerqueira, F.; Sousa, M. E.; Nascimento, M. S. J.; Pinto, N. Bioorg. Med. Chem. 2002, 10, 3725. (d) Schaper, K.-J.; Pickert, M.; Frahm, A. W. Arch. Pharm. Pharm. Med. Chem. 1999, 332, 91. (e) Minami, H.; Kinoshita, M.; Fukuyama, Y.; Komoda, M.; Yoshizawa, T.; Sugiura, M.; Nakagawa, K.; Tago, H. Phytochemistry 1994, $36,501$.

(3) Fukawa, I.; Yoneda, H.; Asahi, K. K. K. K. Eur. Pat. EP0237004, 1987.
(4) Kelkar, A. S.; Letcher, R. M.; Cheung, K.-K.; Chiu, K.-F.; Brown, G. D. J. Chem. Soc., Perkin Trans. 1 2000, 3732.

(5) (a) Cassilas, L. K.; Townsend, C. A. J. Org. Chem. 1999, 64, 4050. (b) Afzal, M.; Al-Hassan, J. M. Heterocycles 1980, 14, 1173. (c) Grover, P. K.; Shah, G. D.; Shah, R. C. J. Chem. Soc. 1955, 3982.

(6) Mahal, H. S.; Venkataraman, K. J. Chem. Soc. 1934, 1767.

(7) Ares, J. J.; Outt, P. E.; Kakodkar, S. V.; Buss, R. C.; Geiger, J. C. J. Org. Chem. 1993, 58, 7903.

(8) Ibrahim, S. S. Ind. Eng. Chem. Res. 2001, 40, 37.

(9) (a) Price, W. A.; Silva, A. M. S.; Cavaleiro, J. A. S. Heterocycles 1993, 36, 2601. (b) Alonso, R.; Brossi, A. Tetrahedron Lett. 1988, 29, 735. (c) Ayyangar, N. R.; Khan, R. A.; Desphande, V. H. Tetrahedron Lett. 1988, 29, 2347.

(10) Typical Experimental Procedure.

Sodium $(0.4 \mathrm{~g}, 16.7 \mathrm{mmol})$ was gradually added to $20 \mathrm{~mL}$ of dry $\mathrm{MeOH}$ and the mixture was stirred until the solution reached r.t. 3-Bromo-2-methylchromone 4 (1 g, $4.2 \mathrm{mmol})$ and the appropriate benzaldehyde $\mathbf{5 a}, \mathbf{b}(5.0 \mathrm{mmol})$ were added and the reaction mixture allowed to stand at r.t. for 48 h. After this period, the solution was poured into ice and $\mathrm{H}_{2} \mathrm{O}$ and the $\mathrm{pH}$ adjusted to 4 with $\mathrm{HCl}$. The yellow solid was removed by filtration, taken up in $\mathrm{CH}_{2} \mathrm{Cl}_{2}$ and purified by silica gel column chromatography using $\mathrm{CH}_{2} \mathrm{Cl}_{2}$ as eluent. The solvent was evaporated to dryness and the residue was recrystallised from EtOH to give the 3-bromo-2-styrylchromones 6a,b in good yields (Table 1).

(11) Physical Data of 4'-Benzyloxy-3-bromo-2-styrylchromone (6b).

Mp 176-178 ${ }^{\circ} \mathrm{C} .{ }^{1} \mathrm{H}$ NMR $\left(300.13 \mathrm{MHz}, \mathrm{CDCl}_{3}\right): \delta=5.09$ (s, $\left.2 \mathrm{H}, 4^{\prime}-\mathrm{OCH}_{2} \mathrm{C}_{6} \mathrm{H}_{5}\right), 6.99$ (d, $\left.2 \mathrm{H}, J=8.8 \mathrm{~Hz}, \mathrm{H}-3^{\prime}, 5^{\prime}\right)$, $7.30(\mathrm{~d}, 1 \mathrm{H}, J=15.8 \mathrm{~Hz}, \mathrm{H}-\alpha), 7.34-7.45(\mathrm{~m}, 6 \mathrm{H}, \mathrm{H}-6$ and $\left.4^{\prime}-\mathrm{OCH}_{2} \mathrm{C}_{6} H_{5}\right), 7.50(\mathrm{~d}, 1 \mathrm{H}, J=7.8 \mathrm{~Hz}, \mathrm{H}-8), 7.56(\mathrm{~d}, 2 \mathrm{H}$, $\left.J=8.8 \mathrm{~Hz}, \mathrm{H}-2^{\prime}, 6^{\prime}\right), 7.62(\mathrm{~d}, 1 \mathrm{H}, J=15.8 \mathrm{~Hz}, \mathrm{H}-\beta), 7.67$ (dt, $1 \mathrm{H}, J=7.8,1.6 \mathrm{~Hz}, \mathrm{H}-7), 8.19(\mathrm{dd}, 1 \mathrm{H}, J=7.9,1.6 \mathrm{~Hz}, \mathrm{H}-$ 5). ${ }^{13} \mathrm{C} \mathrm{NMR}\left(75.47 \mathrm{MHz}, \mathrm{CDCl}_{3}\right): \delta=70.0\left(4^{\prime}-\mathrm{OCH}_{2} \mathrm{C}_{6} \mathrm{H}_{5}\right)$, 108.9 (C-3), $115.3\left(\mathrm{C}-3^{\prime}, 5^{\prime}\right), 116.7$ (C- $\left.\alpha\right), 117.4(\mathrm{C}-8), 122.0$ (C-10), 125.2 (C-6), 126.2 (C-5), 127.4 (C-2,6 of 4'$\left.\mathrm{OCH}_{2} \mathrm{C}_{6} \mathrm{H}_{5}\right), 127.8\left(\mathrm{C}-1^{\prime}\right), 128.1\left(\mathrm{C}-4\right.$ of $\left.4^{\prime}-\mathrm{OCH}_{2} \mathrm{C}_{6} \mathrm{H}_{5}\right)$, $128.6\left(\mathrm{C}-3,5\right.$ of $\left.4^{\prime}-\mathrm{OCH}_{2} \mathrm{C}_{6} \mathrm{H}_{5}\right), 129.7\left(\mathrm{C}-2^{\prime}, 6^{\prime}\right), 133.8(\mathrm{C}-7)$, $136.3\left(\mathrm{C}-1\right.$ of $\left.4^{\prime}-\mathrm{OCH}_{2} \mathrm{C}_{6} \mathrm{H}_{5}\right), 139.2(\mathrm{C}-\beta), 154.7$ (C-9), 158.7 (C-2), $160.6\left(\mathrm{C}-4^{\prime}\right), 172.6(\mathrm{C}-4)$. MS (EI): $\mathrm{m} / \mathrm{z}(\mathrm{rel}$. int. $)=434(14)\left[\mathrm{M}^{+\bullet},{ }^{81} \mathrm{Br}\right], 432(14)\left[\mathrm{M}^{+*},{ }^{79} \mathrm{Br}\right], 388(8), 234$ (7), 205 (8), 92 (15), 91 (100), 65 (9). Anal. Calcd for $\mathrm{C}_{24} \mathrm{H}_{17} \mathrm{BrO}_{3}$ : C, 66.53; H, 3.95. Found: C, 66.48; H, 4.27.

(12) Bigi, F.; Conforti, M. L.; Maggi, R.; Piccinno, A.; Sartori, G. Green Chem. 2000, 2, 101.

(13) (a) Nicolaou, K. C.; Bulger, P. G.; Sarlah, D. Angew. Chem. Int. Ed. 2005, 44, 4442. (b) Farina, V. Adv. Synth. Catal. 2004, 346, 1553. (c) Reetz, M. T.; de Vries, J. G. Chem. Commun. 2004, 1559. (d) Heidenreich, R. G.; Köhler, K.; Krauter, J. G. E.; Pietsch, J. Synlett 2002, 1118. (e) Biffis, A.; Zecca, M.; Basato, M. J. Mol. Catal. A: Chem. 2001, 173, 249. (f) de Meijere, A.; Meyer, F. E. Angew. Chem., Int. Ed. Engl. 1994, 33, 2379.

(14) Typical Experimental Procedure.

A mixture of the appropriate 3-bromo-2-styrylchromone 6a,b $(0.6 \mathrm{mmol}), \mathrm{PPh}_{3}(15.7 \mathrm{mg}, 0.06 \mathrm{mmol})$, tetrakis(triphenylphosphine)palladium $(0)(34.7 \mathrm{mg}, 0.03 \mathrm{mmol})$ and $\mathrm{Et}_{3} \mathrm{~N}(83.6 \mu \mathrm{L}$ or $334.5 \mu \mathrm{L}, 0.6$ or $2.4 \mathrm{mmol})$ in $N$-methyl-2pyrrolidinone $(10 \mathrm{~mL})$ was added to styrenes $7 \mathbf{a}-\mathbf{c}(3 \mathrm{mmol})$. Each reaction was stirred under different conditions of time and temperature according to the substitution of the compounds (Table 2). Then, the mixture was poured into $\mathrm{H}_{2} \mathrm{O}$ and ice and extracted several times with $\mathrm{Et}_{2} \mathrm{O}$ and dried over anhyd $\mathrm{Na}_{2} \mathrm{SO}_{4}$. The solvent was evaporated and the residue taken up in $\mathrm{CH}_{2} \mathrm{Cl}_{2}$ and purified by thin layer 
chromatography (a 7:3 mixture of $\mathrm{CH}_{2} \mathrm{Cl}_{2}$-light $\mathrm{PE}$ as eluent). The product was crystallised from EtOH giving, in each case, the 2,3-diarylxanthones 9a-f (yields described in Table 2).

(15) Physical Data of 2,3-Diphenylxanthone (8a).

$\mathrm{Mp} 152-153^{\circ} \mathrm{C} .{ }^{1} \mathrm{H}$ NMR $\left(300.13 \mathrm{MHz}, \mathrm{CDCl}_{3}\right): \delta=7.18$ $7.29\left(\mathrm{~m}, 10 \mathrm{H}, 2,3-\mathrm{C}_{6} H_{5}\right), 7.41(\mathrm{dt}, 1 \mathrm{H}, J=7.5,1.0 \mathrm{~Hz}, \mathrm{H}-$ 7), $7.53(\mathrm{dd}, 1 \mathrm{H}, J=8.4,0.8 \mathrm{~Hz}, \mathrm{H}-5), 7.57(\mathrm{~s}, 1 \mathrm{H}, \mathrm{H}-4)$, 7.75 (dt, $1 \mathrm{H}, J=7.8,1.6 \mathrm{~Hz}, \mathrm{H}-6), 8.38$ (dd, $1 \mathrm{H}, J=7.9,1.6$ $\mathrm{Hz}, \mathrm{H}-8), 8.38$ (s, $1 \mathrm{H}, \mathrm{H}-1) .{ }^{13} \mathrm{C} \mathrm{NMR}\left(75.47 \mathrm{MHz}, \mathrm{CDCl}_{3}\right.$ ): $\delta=118.0(\mathrm{C}-5), 119.5(\mathrm{C}-4), 120.7$ (C-9a), $121.9(\mathrm{C}-8 \mathrm{a})$, 124.0 (C-7), 126.8 (C-8), 126.9 (C-4'), 127.6 (C-4"), 128.0 $\left(\mathrm{C}-2^{\prime}, 6^{\prime}\right), 128.1\left(\mathrm{C}-3^{\prime \prime}, 5^{\prime \prime}\right), 128.4(\mathrm{C}-1), 129.6\left(\mathrm{C}-2^{\prime \prime}, 6^{\prime \prime}\right)$, $129.9\left(\mathrm{C}-3^{\prime}, 5^{\prime}\right), 134.8$ (C-6), $137.0\left(\mathrm{C}-1^{\prime}\right), 139.8(\mathrm{C}-2), 139.9$ (C-1"), 147.6 (C-3), 155.2 (C-4a), 156.3 (C-4b), 177.0 (C9). MS (EI): $m / z$ (rel. int.) = $348(100)\left[\mathrm{M}^{+*}\right], 347$ (45), 333 (14), 318 (8), 305 (4), 289 (15), 276 (4), 228 (8), 226 (8), 213 (5), 174 (9), 145 (4), 77 (3). HRMS (EI): $\mathrm{m} / \mathrm{z}$ calcd for $\mathrm{C}_{25} \mathrm{H}_{16} \mathrm{O}_{2}$ : 348.1150; found: 348.1158 .

(16) Physical Data of 2,3-Diphenyl-3,4-dihydroxanthone (10a).

${ }^{1} \mathrm{H}$ NMR $\left(300.13 \mathrm{MHz}, \mathrm{CDCl}_{3}\right): \delta=3.01(\mathrm{dd}, 1 \mathrm{H}, J=17.4$, $\left.1.5 \mathrm{~Hz}, \mathrm{H}-4_{\text {trans }}\right), 3.67$ (dd, $\left.1 \mathrm{H}, J=17.4,9.0 \mathrm{~Hz}, \mathrm{H}-4_{\text {cis }}\right), 4.31$ $(\mathrm{dd}, 1 \mathrm{H}, J=9.0,1.5 \mathrm{~Hz}, \mathrm{H}-3), 7.19-7.30(\mathrm{~m}, 8 \mathrm{H}, \mathrm{H}-$ $\left.2^{\prime}, 3^{\prime}, 4^{\prime}, 5^{\prime}, 6^{\prime}, 3^{\prime \prime}, 4^{\prime \prime}, 5^{\prime \prime}\right), 7.32-7.41$ (m, $\left.2 \mathrm{H}, \mathrm{H}-5,7\right), 7.46-7.50$ (m, $\left.2 \mathrm{H}, \mathrm{H}-2^{\prime \prime}, 6^{\prime \prime}\right), 7.56$ (s, $1 \mathrm{H}, \mathrm{H}-1$ ), 7.60 (dt, $1 \mathrm{H}, J=7.8$, $1.6 \mathrm{~Hz}, \mathrm{H}-6), 8.29$ (dd, $1 \mathrm{H}, J=7.9,1.6 \mathrm{~Hz}, \mathrm{H}-8) .{ }^{13} \mathrm{C} \mathrm{NMR}$ $\left(75.47 \mathrm{MHz}, \mathrm{CDCl}_{3}\right): \delta=36.7(\mathrm{C}-4), 41.7(\mathrm{C}-3), 116.8(\mathrm{C}-$ 1), 116.9 (C-9a), 118.0 (C-5), 123.9 (C-8a), 125.1 (C-7), $125.7\left(\mathrm{C}-2^{\prime \prime}, 6^{\prime \prime}\right), 126.2(\mathrm{C}-8), 127.2\left(\mathrm{C}-4^{\prime}\right), 127.4\left(\mathrm{C}-2^{\prime}, 6^{\prime}\right)$, 127.6 (C-4"), $128.5\left(\mathrm{C}-3^{\prime \prime}, 5^{\prime \prime}\right), 129.0\left(\mathrm{C}-3^{\prime}, 5^{\prime}\right), 133.0$ (C-6), 135.3 (C-1"), 139.1 (C-1'), 140.7 (C-2), 155.9 (C-4b), 162.5 $(\mathrm{C}-4 \mathrm{a}), 174.2(\mathrm{C}-9)$. MS $\left(\mathrm{FAB}^{+}\right): \mathrm{m} / \mathrm{z}$ (rel. int. $)=351(37)[\mathrm{M}$ $+\mathrm{H}]^{+}, 212$ (8), 77 (19). HRMS (EI): $\mathrm{m} / z$ calcd for $\mathrm{C}_{25} \mathrm{H}_{18} \mathrm{O}_{2}$ : 350.1307; found: 350.1315 .

(17) (a) Clarke, D. S.; Gabbutt, C. D.; Hepworth, J. D.; Heron, B. M. Tetrahedron Lett. 2005, 46, 5515. (b) de Meijere, A.; Schelper, M.; Knoke, M.; Yucel, B.; Sünnemann, H. W.; Scheurich, R. P.; Arve, L. J. Organomet. Chem. 2003, 687, 249.

(18) Santos, C. M. M.; Silva, A. M. S.; Cavaleiro, J. A. S. unpublished results.
(19) Physical Data of 3-Phenyl-2-(4-methoxyphenyl)xanthone $(\mathbf{8 b})$.

Mp 123-125 ${ }^{\circ} \mathrm{C} .{ }^{1} \mathrm{H}$ NMR $\left(300.13 \mathrm{MHz}, \mathrm{CDCl}_{3}\right): \delta=3.79$ $\left(\mathrm{s}, 3 \mathrm{H}, 4^{\prime}-\mathrm{OCH}_{3}\right), 6.77$ (d, $\left.2 \mathrm{H}, J=8.8 \mathrm{~Hz}, \mathrm{H}-3^{\prime}, 5^{\prime}\right), 7.09$ (d, $\left.2 \mathrm{H}, J=8.8 \mathrm{~Hz}, \mathrm{H}-2^{\prime}, 6^{\prime}\right), 7.20-7.24$ (m, $\left.2 \mathrm{H}, \mathrm{H}-2^{\prime \prime}, 6^{\prime \prime}\right), 7.26-$ $7.30\left(\mathrm{~m}, 3 \mathrm{H}, \mathrm{H}-3^{\prime \prime}, 4^{\prime \prime}, 5^{\prime \prime}\right), 7.39$ (dt, $1 \mathrm{H}, J=7.8,0.9 \mathrm{~Hz}, \mathrm{H}-$ 7), $7.51(\mathrm{~d}, 1 \mathrm{H}, J=8.0 \mathrm{~Hz}, \mathrm{H}-5), 7.53(\mathrm{~s}, 1 \mathrm{H}, \mathrm{H}-4), 7.73$ (dt, $1 \mathrm{H}, J=8.0,1.6 \mathrm{~Hz}, \mathrm{H}-6), 8.34$ (s, $1 \mathrm{H}, \mathrm{H}-1), 8.37$ (dd, $1 \mathrm{H}$, $J=7.8,1.6 \mathrm{~Hz}, \mathrm{H}-8) .{ }^{13} \mathrm{C}$ NMR $\left(75.47 \mathrm{MHz}, \mathrm{CDCl}_{3}\right): \delta=$ $55.2\left(4^{\prime}-\mathrm{OCH}_{3}\right), 113.4\left(\mathrm{C}-3^{\prime}, 5^{\prime}\right), 118.0(\mathrm{C}-5), 119.5(\mathrm{C}-4)$, 120.7 (C-9a), 121.9 (C-8a), 123.9 (C-7), 126.7 (C-8), 127.5 $\left(\mathrm{C}-4^{\prime \prime}\right), 128.1(\mathrm{C}-1), 128.2\left(\mathrm{C}-3^{\prime \prime}, 5^{\prime \prime}\right), 129.6\left(\mathrm{C}-2^{\prime \prime}, 6^{\prime \prime}\right), 131.0$ $\left(\mathrm{C}-2^{\prime}, 6^{\prime}\right), 132.1\left(\mathrm{C}-1^{\prime}\right), 134.7$ (C-6), 136.7 (C-2), 140.0 (C$\left.1^{\prime \prime}\right), 147.5$ (C-3), 155.0 (C-4a), 156.3 (C-4b), 158.6 (C-4'), 177.0 (C-9). MS (EI): $m / z$ (rel. int.) $=378(100)\left[\mathrm{M}^{+*}\right], 363$ (12), 347 (9), 334 (7), 318 (3), 305 (9), 292 (2), 276 (4), 263 (2), 213 (3), 189 (5), 173 (3), 167 (5), 138 (3), 92 (2), 77 (2). Anal. Calcd for $\mathrm{C}_{26} \mathrm{H}_{18} \mathrm{O}_{3}: \mathrm{C}, 82.52 ; \mathrm{H}, 4.79$. Found: $\mathrm{C}$, 82.23; H, 4.94.

(20) Physical Data of 3-(4-Benzyloxyphenyl)-2-(3,4-dimethoxyphenyl)-3,4-dihydroxanthone (10f).

${ }^{1} \mathrm{H}$ NMR (300.13 MHz, $\left.\mathrm{CDCl}_{3}\right): \delta=2.95$ (dd, $1 \mathrm{H}, J=17.3$, $\left.1.5 \mathrm{~Hz}, \mathrm{H}-4_{\text {trans }}\right), 3.61\left(\mathrm{dd}, 1 \mathrm{H}, J=17.3,8.4 \mathrm{~Hz}, \mathrm{H}-4_{\text {cis }}\right), 3.83$ and $3.84\left(2 \mathrm{~s}, 6 \mathrm{H}, 3^{\prime}, 4^{\prime}-\mathrm{OCH}_{3}\right), 4.23(\mathrm{~d}, 1 \mathrm{H}, J=8.3 \mathrm{~Hz}, \mathrm{H}-$ 3), 4.94 (s, $\left.2 \mathrm{H}, 4^{\prime \prime}-\mathrm{OCH}_{2} \mathrm{C}_{6} \mathrm{H}_{5}\right), 6.74(\mathrm{~d}, 1 \mathrm{H}, J=8.5 \mathrm{~Hz}, \mathrm{H}-$ $\left.5^{\prime}\right), 6.84\left(\mathrm{~d}, 2 \mathrm{H}, J=8.7 \mathrm{~Hz}, \mathrm{H}-3^{\prime \prime}, 5^{\prime \prime}\right), 6.97(\mathrm{dd}, 1 \mathrm{H}, J=8.5$, $\left.2.1 \mathrm{~Hz}, \mathrm{H}-6^{\prime}\right), 7.09$ (d, $\left.1 \mathrm{H}, J=2.1 \mathrm{~Hz}, \mathrm{H}-2^{\prime}\right), 7.21$ (d, $2 \mathrm{H}$, $\left.J=8.7 \mathrm{~Hz}, \mathrm{H}-2^{\prime \prime}, 6^{\prime \prime}\right), 7.28-7.39$ (m, $7 \mathrm{H}, \mathrm{H}-5,7$ and H2,3,4,5,6 of 4" $\left.-\mathrm{OCH}_{2} \mathrm{C}_{6} \mathrm{H}_{5}\right), 7.44(\mathrm{~s}, 1 \mathrm{H}, \mathrm{H}-1), 7.57$ (dt, $1 \mathrm{H}$, $J=7.8,1.6 \mathrm{~Hz}, \mathrm{H}-6), 8.28(\mathrm{dd}, 1 \mathrm{H}, J=7.9,1.6 \mathrm{~Hz}, \mathrm{H}-8) .{ }^{13} \mathrm{C}$ $\operatorname{NMR}\left(75.47 \mathrm{MHz}, \mathrm{CDCl}_{3}\right): \delta=36.7(\mathrm{C}-4), 41.1(\mathrm{C}-3), 55.8$ $\left(3^{\prime}, 4^{\prime}-\mathrm{OCH}_{3}\right), 69.9\left(4^{\prime \prime}-\mathrm{OCH}_{2} \mathrm{C}_{6} \mathrm{H}_{5}\right), 108.7\left(\mathrm{C}-2^{\prime}\right), 110.8(\mathrm{C}-$ $\left.5^{\prime}\right), 114.8(\mathrm{C}-1), 115.2\left(\mathrm{C}-3^{\prime \prime}, 5^{\prime \prime}\right), 116.9$ (C-9a), 118.0 (C-5), $118.3\left(\mathrm{C}-6^{\prime}\right), 123.8$ (C-8a), 125.0 (C-7), 126.1 (C-8), 125.7 $\left(\mathrm{C}-2,6\right.$ of $\left.4^{\prime \prime}-\mathrm{OCH}_{2} \mathrm{C}_{6} \mathrm{H}_{5}\right), 127.9\left(\mathrm{C}-4\right.$ of $\left.4^{\prime \prime}-\mathrm{OCH}_{2} \mathrm{C}_{6} \mathrm{H}_{5}\right)$, $128.3\left(\mathrm{C}-2^{\prime \prime}, 6^{\prime \prime}\right), 128.5\left(\mathrm{C}-3,5\right.$ of $\left.4^{\prime \prime}-\mathrm{OCH}_{2} \mathrm{C}_{6} \mathrm{H}_{5}\right), 132.0(\mathrm{C}-$ $\left.1^{\prime}\right), 133.0$ (C-6), 133.1 (C-1"), 135.5 (C-2), 136.9 (C-1 of 4"$\left.\mathrm{OCH}_{2} \mathrm{C}_{6} \mathrm{H}_{5}\right), 148.7$ and $148.8\left(\mathrm{C}-3^{\prime}, 4^{\prime}\right), 155.8(\mathrm{C}-4 \mathrm{~b}), 157.9$ $\left(\mathrm{C}-4^{\prime \prime}\right), 162.3$ (C-4a), 174.2 (C-9). HRMS (EI): $\mathrm{m} / \mathrm{z}$ calcd for $\mathrm{C}_{32} \mathrm{H}_{24} \mathrm{O}_{3}$ : 456.1725; found: 456.1735 .

(21) The relative stereochemistry of protons H-3 and H-4 is referred as cis and trans. 